NBI-HE-95-01

January 1995

\title{
Scaling in quantum gravity
}

\author{
J. Ambjørn and Y. Watabiki \\ The Niels Bohr Institute \\ Blegdamsvej 17, DK-2100 Copenhagen Ø, Denmark
}

\begin{abstract}
The 2-point function is the natural object in quantum gravity for extracting critical behavior: The exponential fall off of the 2-point function with geodesic distance determines the fractal dimension $d_{H}$ of space-time. The integral of the 2-point function determines the entropy exponent $\gamma$, i.e. the fractal structure related to baby universes, while the short distance behavior of the 2-point function connects $\gamma$ and $d_{H}$ by a quantum gravity version of Fisher's scaling relation. We verify this behavior in the case of $2 \mathrm{~d}$ gravity by explicit calculation.
\end{abstract}




\section{Introduction}

Much has been achieved in our understanding of $2 \mathrm{~d}$ quantum gravity, both from the point of view of Liouville theory [1] and from a discretized point of view [2, 3, 4, 5]. However, the simplest and most fundamental concept in gravity, the concept of distance, has only recently been analyzed. In Liouville theory the tool has been the diffusion equation for a random walk on the ensemble of $2 \mathrm{~d}$ manifolds weighted by the Liouville action [6]. In the framework of dynamical triangulations the tool has been the transfer matrix formalism developed in [7]. In this article we show that standard scaling relations known from statistical mechanics follow unambiguously even in quantum gravity once the geodesic distance is used to set the length scale in the problem.

\section{Scaling relations}

Let us define $2 \mathrm{~d}$ quantum gravity as the scaling limit of the so-called simplicial quantum gravity theory. Simplicial quantum gravity can be defined in any dimensions, but we will here restrict ourselves to $2 \mathrm{~d}$. The partition function will be given by:

$$
Z\left(\mu, G_{E}\right)=\sum_{T \in \mathcal{T}} \frac{1}{C_{T}} \mathrm{e}^{-S[T]}
$$

where $S[T]$ is the Einstein-Hilbert action:

$$
S[T]=\mu N_{T}-\frac{1}{4 \pi G_{E}}\left(2-2 g_{T}\right)
$$

In eqs. (11) and (2) $\mathcal{T}$ denotes a suitable class of triangulations of closed 2-manifolds, $T$ a triangulation in $\mathcal{T}, N_{T}$ the number of triangles in $T, C_{T}$ a symmetry factor and $g_{T}$ the genus of the manifold. If we fix the topology, as we will always do in the following, we can drop the last term since it is a topological invariant.

It is known that the partition function $Z(\mu)$ (for a fixed topology) has a critical bare cosmological constant $\mu_{c}$ such that the continuum limit of (国) should be taken for $\mu \rightarrow \mu_{c}$ from above. Define $\triangle \mu \equiv \mu-\mu_{c}$, then

$$
Z(\mu) \sim \text { const. }(\triangle \mu)^{2-\gamma}+\text { less singular terms }
$$

For 2 d gravity $\gamma=-1 / 2$ for spherical topology. In the following we will consider only triangulations with spherical topology.

We define the geodesic distance between two links as the shortest path of links connecting the two links in the dual lattice, i.e. the shortest "triangle-path" between the two links on the original triangulation. Let us by $\mathcal{T}_{2}(r)$ denote the ensemble of

\footnotetext{
${ }^{1}$ It is convenient here to use the geodesic distance between links rather than between vertices. As will be clear later the results are independent of this definition. We choose it because it is technically convenient in the analytic calculations.
} 


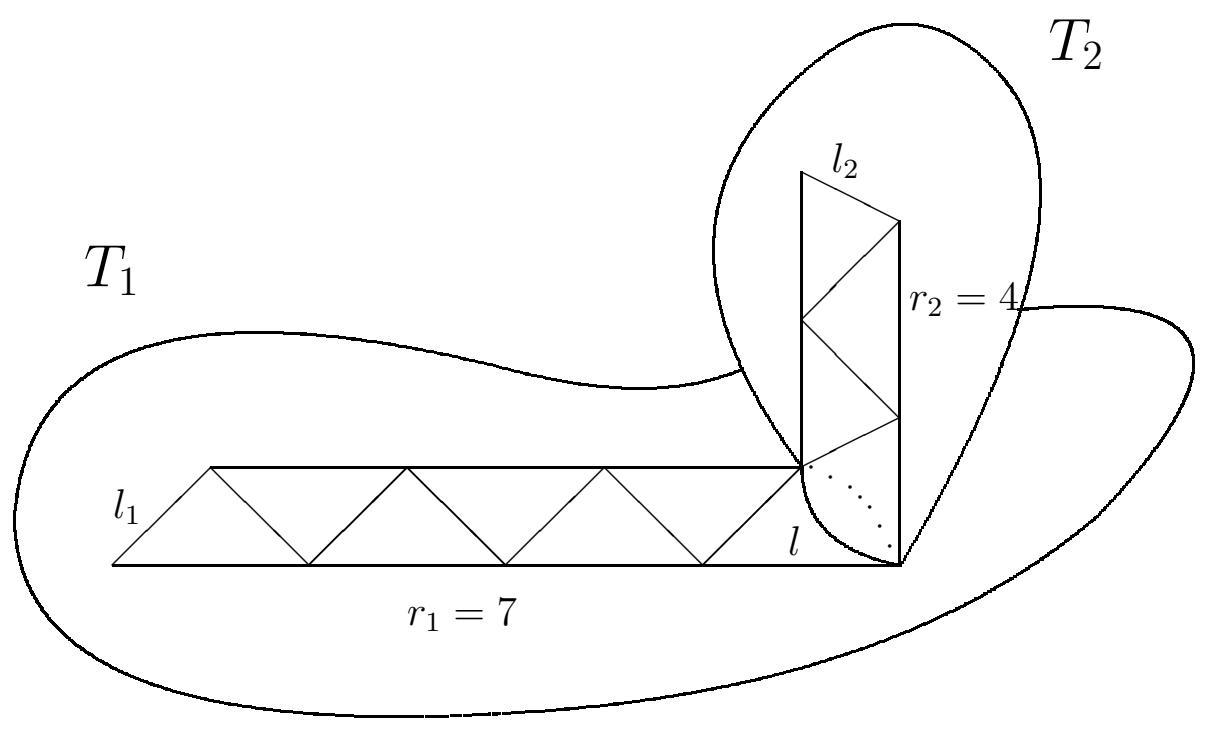

Figure 1: The inequality (6). Two triangulations with marked links separated by distances $r_{1}$ and $r_{2}$ can be glued together to a triangulation where the marked links has a distance $r_{1}+r_{2}$ but the same number of triangles by cutting open a marked link in each of the triangulations to a 2-loop boundary and glue together the two boundaries.

(spherical) triangulations with two marked links separated a geodesic distance $r$ (we assume for the moment that the link length is $a=1$ ). We can now define the 2-point function of quantum gravity by

$$
G_{\mu}(r)=\sum_{T \in \mathcal{T}_{2}(r)} \mathrm{e}^{-\mu N_{T}}
$$

The 2-point function falls off exponentially for $r \rightarrow \infty$.

$$
\lim _{r \rightarrow \infty} \frac{-\log G_{\mu}(r)}{r}=m(\triangle \mu) \geq 0 .
$$

This trivial but important relation follows from the fact that

$$
G_{\mu}\left(r_{1}+r_{2}\right) \geq G_{\mu}\left(r_{1}\right) G_{\mu}\left(r_{2}\right)
$$

simply because each term on the rhs of eq. (6) can be given an interpretation as a term belonging to the lhs of eq. (6). This is illustrated in fig. 1. Eq. (6) shows that $-\log G_{\mu}(r)$ is sub-additive and this ensures the existence of the limit (5). In addition $m(\Delta \mu) \geq 0$ because $G_{\mu}(r)$ is a decreasing function of $r$. Again this follows from general arguments which allow us to bound the number of triangulations with $N_{T}$ triangles and two marked links separated a distance $r$ in terms of the number of triangulations with $N_{T}$ triangles and two marked links separated a distance $r^{\prime}<r$. 
The same kind of arguments lead to the conclusion that $m^{\prime}(\Delta \mu)>0$ for $\Delta \mu>0$, i.e. $m(\triangle \mu)$ is a decreasing function as $\mu \rightarrow \mu_{c}$.

Similarly we can define $\mathcal{T}\left(l_{1}, l_{2} ; r\right)$ as the class of triangulations with an entrance boundary loop $l_{1}$ (with one marked link) and an exit boundary loop $l_{2}$, separated a geodesic distance $r$. $l_{1}$ and $l_{2}$ are the number of links at the entrance boundary loop and that at the exit boundary loop, respectively. We say that $l_{1}$ and $l_{2}$ are separated by a geodesic distance $r$ if all links $l \in l_{2}$ has the geodesic distance $r$ to $l_{1}$. Finally the geodesic distance between a link $l$ and a set of links (like the loop $l_{1}$ ) is the minimum of the geodesic distances between the link $l$ and the links in the set. We define ${ }^{2}$

$$
G_{\mu}\left(l_{1}, l_{2} ; r\right)=\sum_{T \in \mathcal{T}\left(l_{1}, l_{2} ; r\right)} \mathrm{e}^{-\mu N_{T}} .
$$

The 2-loop functions fall off exponentially for $r \rightarrow \infty$. Again this follows from the subadditivity argument since one has [7, 8]:

$$
G_{\mu}\left(l_{1}, l_{2} ; r_{1}+r_{1}\right)=\sum_{l=1}^{\infty} G_{\mu}\left(l_{1}, l ; r_{1}\right) G_{\mu}\left(l, l_{2} ; r_{2}\right) \text {. }
$$

It is easy to show, by arguments identical to the ones used originally for random surfaces on hypercubic lattices [9], that the mass is defined by the exponential decay of the two-loop function is independent of the length of the boundary loops and consequently identical to the mass defined by the 2-point function.

The important point is that the "mass" $m(\triangle \mu)$ dictates the scaling in quantum gravity. We can view $G_{\mu}(r)$ as the partition function for universes of linear extension $r$ and in order that this partition function survives in the continuum limit it is necessary that we have

$$
m(\triangle \mu) r=M R
$$

where $M$ and $R$ are kept fixed in the continuum limit where the number of lattice steps $r$ goes to infinity. There can only be a continuum limit if $m(\triangle \mu) \rightarrow 0$ for $\Delta \mu \rightarrow 0$. In addition almost all critical properties of quantum gravity can be read off directly from this function in the scaling limit. This has already been emphasized in the more general context of higher dimensional quantum gravity [10] and more specifically in two dimensions 111 (where an explicit solution was given for a toy model of branched polymers), but it is worth to repeat the arguments. First one would in general expect the exponential decay of $G_{\mu}(r)$ to be replaced by a power fall off when $m(\triangle \mu)=0$, or more precisely in the region where $1 \ll r \ll 1 / m(\triangle \mu)$. The behavior $G_{\mu}(r)$ is is assumed to be:

$$
\begin{array}{llrl}
G_{\mu}(r) & \sim \mathrm{e}^{-m(\Delta \mu) r} & & \text { for } m(\triangle \mu) r \gg 1 \\
G_{\mu}(r) \sim r^{1-\eta} & & \text { for } 1 \ll r \ll \frac{1}{m(\triangle \mu)}
\end{array}
$$

\footnotetext{
${ }^{2}$ Sometimes a different notation is used and the entrance loop is unmarked while the exit loop is marked. The difference in these functions will be some trivial factors of $l: l_{2} G_{\mu}\left(l_{1}, l_{2} ; r\right)=$ $l_{1} G_{\mu}^{\prime}\left(l_{1}, l_{2} ; r\right)$ where $G^{\prime}$ denotes the 2-loop function where the exit loop is marked.
} 


$$
\chi_{\mu} \equiv \int d r G_{\mu}(r) \sim \frac{\partial^{2} Z(\mu)}{\partial \mu^{2}} \sim \operatorname{const} .(\triangle \mu)^{-\gamma} .
$$

These are standard definitions in statistical mechanics. The exponent $\eta$ is called the anomalous scaling dimension since a free propagator in any space-time dimensions has $\eta=0$. This follows by integrating the usual free propagator over the angular variables corresponding to a fixed value of $r$.

Two scaling relations follow directly from the definitions if we assume that $m(\triangle \mu) \rightarrow 0$ for $\triangle \mu \rightarrow 0$ as:

$$
m(\triangle \mu) \sim(\triangle \mu)^{\nu}
$$

From eq. (12) it follows, after differentiating a sufficient number of times after $\mu$ that

$$
\gamma=\nu(2-\eta)
$$

a relation known in statistical mechanics as Fisher's scaling relation. In that case it will typically be a relation between the spin susceptibility exponent $\gamma$, the critical exponent $\nu$ of the spin-spin correlation length and the anomalous scaling exponent of the spin-spin correlation function. It is remarkable that it is still valid in quantum gravity. The other scaling relation is

$$
\nu=\frac{1}{d_{H}}
$$

where $d_{H}$ denotes the (internal) Hausdorff dimension of the ensemble of random surfaces given by eq. (4). To be more precise we define the (internal) Hausdorff dimension of this ensemble by

$$
\langle N\rangle_{r} \sim r^{d_{H}}, \quad r \rightarrow \infty, \quad m(\triangle \mu) r=\text { const. }
$$

where

$$
\langle N\rangle_{r} \equiv \frac{\sum_{T \in \mathcal{T}_{2}(r)} N_{T} \mathrm{e}^{-\mu N_{T}}}{\sum_{T \in \mathcal{T}_{2}(r)} \mathrm{e}^{-\mu N_{T}}} .
$$

It follows from the definitions that:

$$
\langle N\rangle_{r} \sim-\frac{1}{G_{\mu}(r)} \frac{\partial G_{\mu}(r)}{\partial \mu} \sim m^{\prime}(\triangle \mu) r \sim r^{1 / \nu}
$$

It is interesting to give a direct physical interpretation of the short distance behavior of the $G_{\mu}(r)$ as defined by (11). In order to do so let us change from the grand canonical ensemble given by (4) to the canonical ensemble defined by

$$
G(r, N)=\sum_{T \in \mathcal{T}_{2}(r, N)} 1
$$

where $\mathcal{T}_{2}(r, N)$ denotes the triangulations which $N$ triangles and two marked links separated a distance $r$. $G(r, N)$ is the 2-point function where the number of triangles 
is fixed to $N$. For $r=0$ we have the following $N$ dependence (for the $r$ dependence see (25) below)

$$
G(0, N) \sim N^{\gamma-2} \mathrm{e}^{\mu_{c} N}
$$

The reason is that the partition function for a finite volume $N$ is assumed to behave like

$$
Z(N) \sim N^{\gamma-3} \mathrm{e}^{\mu_{c} N}
$$

The 1-point function is for large $N$ proportional to $N Z(N)$ since it counts the triangulations with one marked link or triangle or vertex depending on the precise definition, and for $r=0$ (or just small) there is essntially no difference between the 1-point function and $G(0, N)$.

$G(r, N)$ is related to $G_{\mu}(r)$ by a (discrete) Laplace transformation:

$$
G_{\mu}(r)=\sum_{N} G(r, N) \mathrm{e}^{-\mu N}
$$

The long distance behavior of $G(r, N)$ is determined by the long distance behavior of $G_{\mu}(r)$. Close to the scaling limit it follows by direct calculation (e.g. a saddlepoint calculation) that

$$
\begin{aligned}
G_{\mu}(r) & \sim \mathrm{e}^{-r(\triangle \mu)^{1 / d_{H}}} \Rightarrow \\
G(r, N) & \sim \mathrm{e}^{-c\left(r^{d_{H}} / N\right)^{\frac{1}{d_{H}-1}}} \mathrm{e}^{\mu_{c} N} \quad \text { for } \quad r^{d_{H}}>N,
\end{aligned}
$$

where $c=\left(d_{H}-1\right) / d_{H}^{d_{H} /\left(d_{H}-1\right)}$.

On the other hand the short distance behavior of $G_{\mu}(r)$ is determined by the short distance behavior of $G(r, N)$ which is simple. Eqs. (16) and (17) defined the concept of Hausdorff dimension in the grand canonical ensemble. A definition in the canonical ensemble would be: Take $N^{1 / d_{H}} \gg r$ and simply count the volume (here number of triangles) of a "spherical shell" of thickness 1 and radius $r$ from a marked link, sum over all triangulations with one marked link and $N$ triangles, and divide by the total number of triangulations with one marked link and $N$ triangles. Call this number $\langle n(r)\rangle_{N}$. The Hausdorff dimension is then defined by

$$
\langle n(r)\rangle_{N} \sim r^{d_{H}-1} \quad \text { for } \quad 1 \ll r \ll N^{1 / d_{H}} .
$$

It follows from the definitions that we can write

$$
\begin{aligned}
\langle n(r)\rangle_{N} & \sim \frac{G(r, N)}{G(0, N)}, \quad \text { i.e } \\
G(r, N) & \sim r^{d_{H}-1} N^{\gamma-2} \mathrm{e}^{\mu_{c} N} \text { for } 1 \ll r \ll N^{1 / d_{H}} .
\end{aligned}
$$

We can finally calculate the short distance behavior of $G_{\mu}(r)$ from eq. (22). From (23) the sum is cut off at $N \sim r^{d_{H}}$. For $\mu \rightarrow \mu_{c}$, i.e. $\triangle \mu$ small we get:

$$
G_{\mu}(r) \sim r^{d_{H}-1} \sum_{N=1}^{r^{d_{H}}} N^{\gamma-2} \sim r^{\gamma d_{H}-1}
$$


This is actually an independent derivation of Fisher's scaling relation since it shows directly that $\eta=2-\gamma d_{H}$, and it has the advantage that it gives a physical interpretation of the anomalous scaling dimension $\eta$. In addition it proves that the canonical and grand canonical definition of Hausdorff dimension in fact agrees.

The model of branched polymers $(B P)$ provides us with a simple, but non-trivial example of the above scenario [11]. Here we will define branched polymers as the sum over all tree graphs (no loops in the graphs) with certain weights given to the graphs according to the following definition of the partition function:

$$
Z(\mu)=\sum_{B P} \frac{1}{C_{B P}} \rho(B P) \mathrm{e}^{-\mu|B P|},
$$

where $|B P|$ is the number of links in a $B P$ and $\mu$ is a chemical potential for the number of links, while

$$
\rho(B P)=\prod_{i \in B P} f\left(n_{i}\right),
$$

where $i$ denotes a vertex, $n_{i}$ the number of links joining at vertex $i$ and $f\left(n_{i}\right)$ is non-negative. $f\left(n_{i}\right)$ can be viewed as the unnormalized branching weight for one link branching into $n_{i}-1$ links at vertex $i$. Finally $C_{B P}$ is a symmetry factor such that rooted branched polymers, i.e. polymers with the first link marked, is counted only once.

This model can be solve [4, 11. It has a critical point $\mu_{c}$ (depending on $f$ ) and close to the critical point we have:

$$
Z^{\prime \prime}(\mu) \sim(\triangle \mu)^{-1 / 2}, \quad \triangle \mu \equiv \mu-\mu_{c}
$$

i.e. $\gamma=1 / 2$ for branched polymers. On the branched polymers we define the "geodesic distance" between two vertices as the shortest link path, which is unique since we consider tree-graphs. The graphical representation of the 2-point function is show in fig. 2. Had it not been for the ability to branch, the 2-point function would simply be

$$
G_{\mu}(r)=\mathrm{e}^{-\mu r} .
$$

However, the insertion of 1-point functions at any vertex leads to a non-analytic coupling constant renormalization and the result is changed to [11]

$$
G_{\mu}(r)=\text { const. } \mathrm{e}^{-\kappa r \sqrt{\triangle \mu}} \text { for } \triangle \mu \rightarrow 0,
$$

where $\kappa$ is some positive constants depending on $f$. We can now find $G(r, N)$ by an inverse Laplace transformation:

$$
G(r, N)=\text { const. } N^{-3 / 2} r \mathrm{e}^{-\kappa^{2} r^{2} / 4 N} .
$$

We confirm from this explicit expression that the (internal) Hausdorff dimension of branched polymers is 2 (like a smooth surface !) and that $\gamma=1 / 2$ since the prefactor of $G(r, N)$ for small $r$ should be $N^{\gamma-2} r^{d_{H}-1}$.

It should be emphasized again that these definitions and scaling relations are valid for simplicial gravity in three and four dimensions as defined in [10, 12, 13]. In the rest of this paper we will study how they are realized in $2 \mathrm{~d}$ simplicial quantum gravity where the exact solution can be found. 


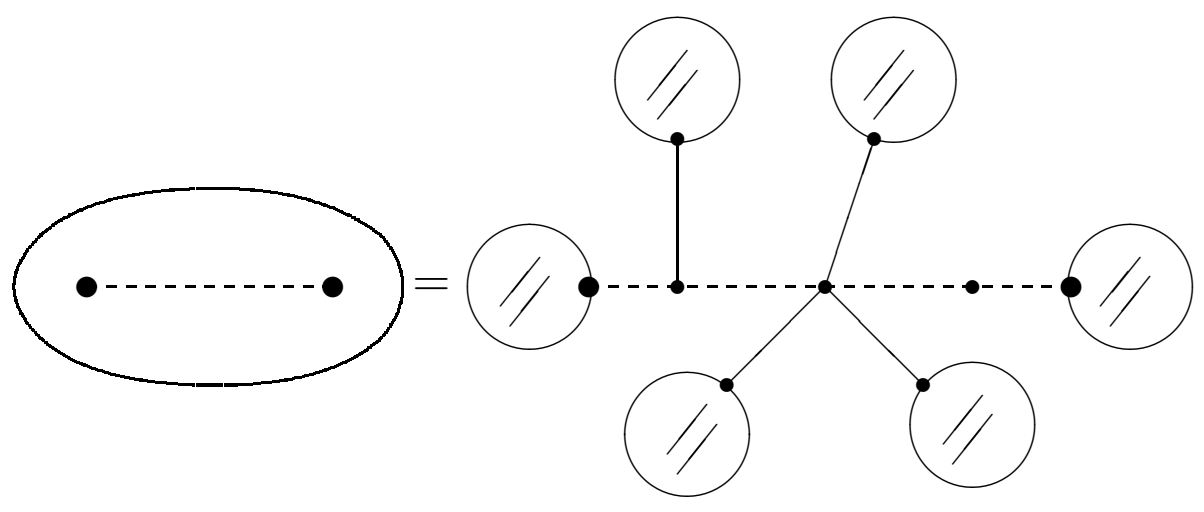

Figure 2: The graphical representation of the 2-point function for branched polymers. The dashed line represents the unique shortest path between the two marked vertices. The "blobs" represent the contribution from all rooted polymers branching out from a vertex.

\section{The 2-point function}

Let us first define the generating function for 2-loop amplitudes (7) by:

$$
G_{\mu}(x, y ; r)=\sum_{l_{1}, l_{2}=1}^{\infty} x^{l_{1}} y^{l_{2}} G_{\mu}\left(l_{1}, l_{2} ; r\right) .
$$

We can reconstruct $G_{\mu}\left(l_{1}, l_{2} ; r\right)$ by

$$
G_{\mu}\left(l_{1}, l_{2} ; r\right)=\oint_{C_{x}} \frac{d x}{2 \pi \mathrm{i} x} x^{-l_{1}} \oint_{C_{y}} \frac{d y}{2 \pi \mathrm{i} y} y^{-l_{2}} G_{\mu}(x, y ; r),
$$

where the contours $C_{x}$ and $C_{y}$ surround the origin and avoid the cuts of $G_{\mu}(x, y ; r)$. The fundamental composition law (8) reads:

$$
G_{\mu}\left(x, y ; r_{1}+r_{2}\right)=\oint_{C} \frac{d z}{2 \pi \mathrm{i} z} G_{\mu}\left(x, \frac{1}{z} ; r_{1}\right) G_{\mu}\left(z, y ; r_{2}\right)
$$

The boundary condition to be imposed is that

$$
G_{\mu}\left(l_{1}, l_{2} ; r=0\right)=\delta_{l_{1}, l_{2}} \quad \text { or } \quad G_{\mu}(x, y ; r=0)=\frac{x y}{1-x y} .
$$

The important insight obtained in [7, 8] is that the 2-loop function satisfies a simple differential equation. Using the so-called peeling decomposition defined in [8] the differential equation has the form [8],

$$
\frac{\partial}{\partial r} G_{\mu}(x, y ; r)=x \frac{\partial}{\partial x}\left(2 x^{2} f_{\mu}(x) G_{\mu}(x, y ; r)\right)
$$

which gives the same differential equation in the limit $\triangle \mu \rightarrow 0$ as was obtained by combinatorial arguments in [7]. 
Let $F_{\mu}(x)$ denote the generating functional for 1-loop functions with one marked link:

$$
F_{\mu}(x)=\sum_{l} x^{l} F_{\mu}(l)
$$

It is well known that

$$
\begin{aligned}
F_{\mu}(x) & =\frac{1}{2}\left(\frac{1}{x^{2}}-\frac{g}{x^{3}}\right)+f_{\mu}(x), \quad g \equiv \mathrm{e}^{-\mu}, \\
f_{\mu}(x) & =\frac{g}{2 x}\left(\frac{1}{x}-c_{2}\right) \sqrt{\left(\frac{1}{x}-c_{1}\right)\left(\frac{1}{x}-c_{0}\right)},
\end{aligned}
$$

where $c_{0}<0<c_{1}<c_{2}$ as long as $\mu>\mu_{c}$. The only thing we need to know is that at the critical point $\mu_{c}$ we have $c_{2}=c_{1}$, (which we denote $1 / x_{c}$ ) and away from the critical point

$$
\begin{aligned}
& c_{2}(\mu)=1 / x_{c}+\frac{\alpha}{2} \sqrt{\triangle \mu}+\mathcal{O}(\triangle \mu) \\
& c_{1}(\mu)=1 / x_{c}-\alpha \sqrt{\triangle \mu}+\mathcal{O}(\triangle \mu) \\
& c_{0}(\mu)=c_{0}\left(\mu_{c}\right)+\mathcal{O}(\triangle \mu)
\end{aligned}
$$

Here $\alpha$ is a positive constant p $^{\text {of }}$ order $\mathcal{O}(1)$ and the scaling limit is obtained when $x=x_{c}-\mathcal{O}(\sqrt{\triangle \mu})$. In this region it is seen that

$$
f_{\mu}(x) \sim(\triangle \mu)^{3 / 4}
$$

and this is the reason the difference equation originating from (35) with $r_{1}=1$ can be replaced with a differential equation for $\Delta \mu \rightarrow 0$ even if $r$ is discrete.

The solution to (36) and (37) is:

$$
G_{\mu}(x, y ; r)=\frac{\hat{x}^{2} f_{\mu}(\hat{x})}{x^{2} f_{\mu}(x)} \frac{\hat{x} y}{1-\hat{x} y}
$$

Here $\hat{x}(x, r)$ is the solution to the characteristic equation of the partial differential equation (37). The integral of the characteristic equation is

$$
r=\int_{x}^{\hat{x}(x, r)} \frac{d x^{\prime}}{2 x^{\prime 3} f_{\mu}\left(x^{\prime}\right)}=\left[\frac{1}{\delta_{0}} \sinh ^{-1} \sqrt{\frac{\delta_{1}}{1-c_{2} x^{\prime}}-\delta_{2}}\right]_{x^{\prime}=x}^{x^{\prime}=\hat{x}(x, r)},
$$

and this expression can be by inverted to give:

$$
\hat{x}(x, r)=\frac{1}{c_{2}}-\frac{\delta_{1}}{c_{2}} \frac{1}{\sinh ^{2}\left(\delta_{0} r+\sinh ^{-1} \sqrt{\left.\frac{\delta_{1}}{1-c_{2} x}-\delta_{2}\right)}+\delta_{2}\right.},
$$

\footnotetext{
${ }^{3}$ The values of the constants which enter are as follows: $\mathrm{e}^{-\mu_{c}}=g_{c}=1 /\left(2 \cdot 3^{\frac{3}{4}}\right), x_{c}=\frac{1}{2}\left(3^{\frac{1}{4}}-3^{-\frac{1}{4}}\right)$, $c_{0}\left(\mu_{c}\right)=3^{\frac{3}{4}}\left(1-3^{\frac{1}{2}}\right)$ and $\alpha=4 \cdot 3^{-\frac{1}{4}}$.
} 
where $\delta_{0}, \delta_{1}$ and $\delta_{2}$ are all positive and defined by

$$
\begin{aligned}
\delta_{0} & =\frac{g}{2} \sqrt{\left(c_{2}-c_{1}\right)\left(c_{2}-c_{0}\right)}=\mathcal{O}\left((\triangle \mu)^{\frac{1}{4}}\right), \\
\delta_{1} & =\frac{\left(c_{2}-c_{1}\right)\left(c_{2}-c_{0}\right)}{c_{2}\left(c_{1}-c_{0}\right)}=\mathcal{O}(\sqrt{\triangle \mu}), \\
\delta_{2} & =-\frac{c_{0}\left(c_{2}-c_{1}\right)}{c_{2}\left(c_{1}-c_{0}\right)}=\mathcal{O}(\sqrt{\triangle \mu}) .
\end{aligned}
$$

It is readily checked that $\hat{x} \rightarrow 1 / c_{2}$ for $r \rightarrow \infty$ and $\hat{x}(x, r=0)=x$. In principle we can calculate $G_{\mu}\left(l_{1}, l_{2} ; r\right)$ from eqs. (34), (45) and (47). Let us only here verify that the exponential decay of $G_{\mu}\left(l_{1}, l_{2} ; r\right)$ is independent of $l_{1}$ and $l_{2}$. For $r \rightarrow \infty$ one gets

$$
G_{\mu}\left(l_{1}, l_{2} ; r\right)=\text { const. } \delta_{0} \delta_{1} \mathrm{e}^{-2 \delta_{0} r}+\mathcal{O}\left(\mathrm{e}^{-4 \delta_{0} r}\right)
$$

where const. is a function of order $\mathcal{O}(1)$ which depends on $c_{0}, c_{1}, c_{2}, l_{1}$ and $l_{2}$.

We can express the 2-point function $G_{\mu}(r)$ in terms of the 2-loop function and the 1-loop function. Let us consider a marked link. For a given triangulation we can systematically work our way out to the links having a distance $r$ from the marked link by peeling off layers of triangles having the distances $1,2, \ldots, r$ to the marked link. After $r$ steps we have a boundary consisting of a number of disconnected boundary loops, all with a distance $r$ to the marked link. One of these is the exit loop described by the 2-loop function and we get the 2-point function by closing the exit loop of length $l_{2}$ by multiplying the 2-loop function $G_{\mu}\left(l_{1}=1, l_{2} ; r\right)$ by the 1-loop function $F_{\mu}\left(l_{2}\right)$ f and the perform the sum over $l_{2}$, i.e., as shown in fig. 3,

$$
\begin{aligned}
G_{\mu}(r) & =\sum_{l_{2}=1}^{\infty} G_{\mu}\left(l_{1}=1, l_{2} ; r\right) l_{2} F_{\mu}\left(l_{2}\right) \\
= & \left.\frac{\partial}{\partial x} \oint_{C_{y}} \frac{d y}{2 \pi \mathrm{i} y} G_{\mu}\left(x, \frac{1}{y} ; r\right) y \frac{\partial}{\partial y} F_{\mu}(y)\right|_{x=0} \\
= & \left.\frac{\partial}{\partial x} F_{\mu}(\hat{x})\right|_{x=0}=\left.\frac{1}{g} \frac{\partial}{\partial r} F_{\mu}(\hat{x})\right|_{x=0} .
\end{aligned}
$$

As long as $c_{2}-c_{1}$ is small and $r$ is larger than a few lattice spacings, we get:

$$
G_{\mu}(r)=\text { const. } \delta_{0} \delta_{1} \frac{\cosh \left(\delta_{0} r\right)}{\sinh ^{3}\left(\delta_{0} r\right)}\left(1+\mathcal{O}\left(\delta_{0}\right)\right) .
$$

Formula (53) shows how to take the scaling limit: Let us return to the original formulation and write in the limit $\triangle \mu \rightarrow 0$ :

$$
G_{\mu}(r)=\text { const. }(\triangle \mu)^{3 / 4} \frac{\cosh \left[(\triangle \mu)^{\frac{1}{4}} \beta r\right]}{\sinh ^{3}\left[(\triangle \mu)^{\frac{1}{4}} \beta r\right]},
$$

where const. and $\beta$ are positive constants of order $\mathcal{O}(1)\left(\beta=\sqrt{6} g_{c}\right)$.

We conclude the following:

\footnotetext{
${ }^{4}$ To be more precise we have to multiply the 2-loop function $G_{\mu}\left(l_{1}, l_{2} ; r\right)$ by $l_{2}$ since the exit loop is unmarked and we can glue the marked one-loop cap to it in $l_{2}$ ways.
} 


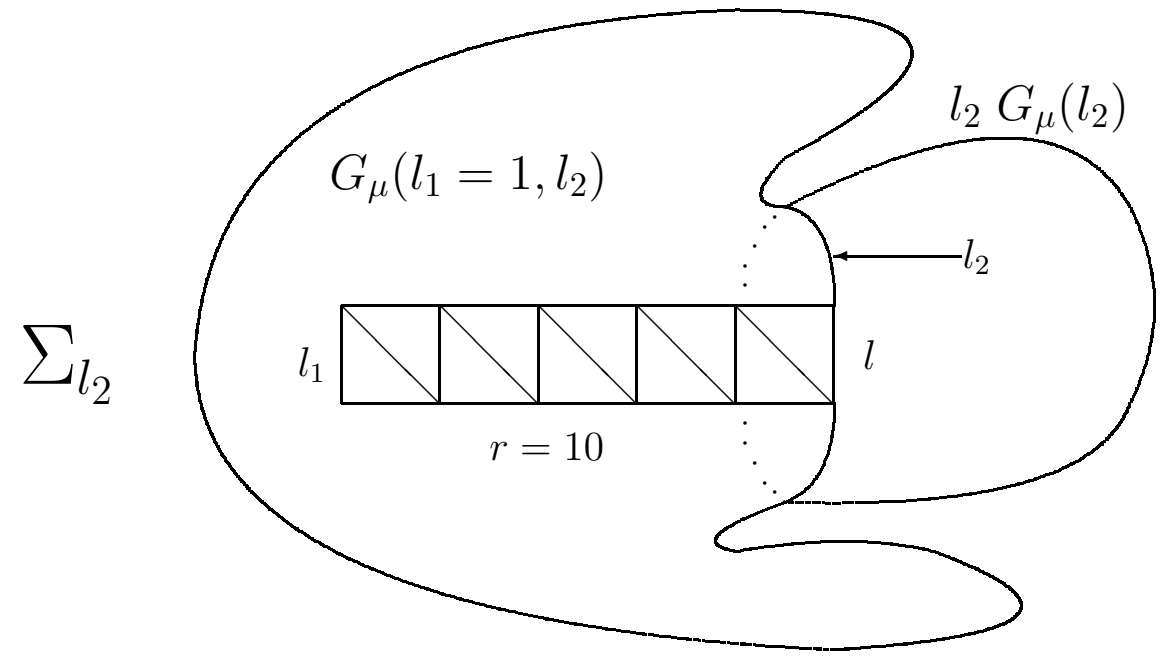

Figure 3: The 2-point function represented as a summation over 2-loop functions times 1-loop functions.

1. $G_{\mu}(r)$ falls of like $\mathrm{e}^{-2(\Delta \mu)^{\frac{1}{4}} \beta r}$ for $r \rightarrow \infty$, i.e. the critical exponent $\nu=\frac{1}{4}$ and the Hausdorff dimension $d_{H}=4$.

2. $G_{\mu}(r)$ behaves like $r^{-3}$ for $1 \ll r \ll \Delta \mu^{-\frac{1}{4}}$, i.e. the scaling exponent $\eta=4$.

3. From Fisher's scaling relation we get $\gamma=\nu(2-\eta)=-1 / 2$. This well known result can of course also be derived directly from

$$
\chi_{\mu}=\sum_{r=1}^{\infty} G_{\mu}(r)=\text { const. }-c^{2}(\triangle \mu)^{\frac{1}{2}}+\cdots
$$

by use of (54), but it should be clear that the explicit calculation in (55) is nothing but a specific example of the general calculation used in proving Fisher's scaling relation. What is somewhat unusual compared to ordinary statistical systems is that the anomalous scaling dimension $\eta>2 . \eta=0$ is the ordinary free field result, while $\eta=2$ is the infinite temperature limit, and for statistical systems we expect $\eta<2$. A final comment to (55) is that the contant in front of $(\triangle \mu)^{\frac{1}{2}}$ is negative, as indicated by the notation. This has a direct physical interpretation: $G_{\mu}(r)$ is by definition positive and the same is the case for $\chi_{\mu}$. However, since $\gamma=-1 / 2$ it follows that $\chi$ will not be divergent at the critical point $\mu_{c}$. But

$$
\tilde{\chi}_{\mu} \equiv-\frac{d \chi_{\mu}}{d \mu} \sim \frac{c^{2}}{(\triangle \mu)^{\frac{1}{2}}}+\cdots
$$

is divergent for $\mu \rightarrow \mu_{c}$ and has to be positive since it, close to the scaling limit, has the interpretation as the sum over all triangulations with three marked links. 
4. Any 2-loop function $G_{\mu}\left(l_{1}, l_{2} ; r\right)$ has the same behavior as $G_{\mu}(r)$ as long as $l_{1}, l_{2}$ stay finite as $\triangle \mu \rightarrow 0$.

It is clear that we could have taken the continuum limit almost at any point in the above calculations, and in fact already in the basic equation (37) by the substitution:

$$
\begin{gathered}
\frac{1}{x}=\frac{1}{x_{c}}+\alpha \xi a, \quad R=\beta r \sqrt{a}, \quad \triangle \mu=\mu_{r} a^{2} \\
f_{\mu}(x) \sim a^{3 / 2} \mathcal{F}_{\mu_{r}}(\xi)+\mathcal{O}\left(a^{5 / 2}\right), \quad \mathcal{F}_{\mu_{r}}(\xi)=\left(\xi-\frac{1}{2} \sqrt{\mu_{r}}\right) \sqrt{\xi+\sqrt{\mu_{r}}}
\end{gathered}
$$

where $\mathcal{F}_{\mu_{r}}(\xi)$ is the universal disk-amplitude [14, 15]. The reason we kept the discretized version thoughout the calculation was to avoid any ambiguity in going from the 2-loop function to the 2-point function. Properties of the continuum 2-loop function have already been studied [7, 8, 16], but in the continuum version the length of the bounday loops $l_{i}$ is already taken to infinity by $L=l a, a \rightarrow 0, l \rightarrow \infty, L$ fixed. To get the 2-point function we would have to take the limit $L_{1} \rightarrow 0$ in the continuum version $G_{\mu_{r}}\left(L_{1}, L_{2} ; R\right)$ of $G_{\mu}\left(l_{1}, l_{2} ; r\right)$. We avoid this ambiguity and can write directly for the continuum 2-point function:

$$
G_{\mu_{r}}(R)=\lim _{a \rightarrow 0}(\sqrt{a})^{\eta-1} G_{\mu}(r) \sim\left(\mu_{r}\right)^{3 / 4} \frac{\cosh \left[\left(\mu_{r}\right)^{\frac{1}{4}} R\right]}{\sinh ^{3}\left[\left(\mu_{r}\right)^{\frac{1}{4}} R\right]} .
$$

The factor in front of $G_{\mu}(r)$ is the usual "wave function renormalization" present in the path integral representation of the propagator. In this way the "mass" (9) $M=2 \mu_{r}^{1 / 4}$, the unusual power due to $d_{H}=4$. Again we find by explicit calculation the continuum version of (55)

$$
\chi_{\mu_{r}}=\int_{0}^{\infty} d R G_{\mu_{r}}(R) \sim \frac{\text { const. }}{a}-\frac{1}{6} \mu_{r}^{1 / 2}
$$

where the constant in front of $\mu_{r}^{\frac{1}{2}}$ has to be negative for the reasons mentioned above.

It is interesting to note that the zero of $f_{\mu}(x)\left(\right.$ or $\left.\mathcal{F}_{\mu_{r}}(\xi)\right)$ determines the infinite $r$ limit of the 2-loop (or 2-point) function. This follows directly from the solution (46) to the characteristic equation for (37). The distance $r$ can only diverge if $\hat{x} \rightarrow 1 / c_{2}$, the zero of $f_{\mu}(x)$. This zero is usually uniquely determined by the requirement that the generating functional $F_{\mu}(x)$ is analytical away from a cut on the real axis and goes to a constant for $|x| \rightarrow 0$. We now see a direct physical interpretation: The zero of $f_{\mu}(x)$, or more suggestive: the pole of $1 / f_{\mu}(x)$, determines the mass of the 2-point function.

Recall that for the branched polymer model we found that the 2-point function is $G_{\mu}^{(B P)}(r) \sim \mathrm{e}^{-\kappa \sqrt{\triangle \mu} r}$, or introducing continuous variables $\sqrt{\triangle \mu}=M a$ and $R=\kappa r a$ :

$$
G_{M}^{(B P)}(R)=\mathrm{e}^{-M R}
$$


and compare this to the $2 \mathrm{~d}$ quantum gravity 2-point function (59):

$$
G_{M}(R)=\frac{1}{8} M^{3} \frac{\cosh M R / 2}{\sinh ^{3} M R / 2}=\frac{1}{2} M^{3} \sum_{n=1}^{\infty} n^{2} \mathrm{e}^{-n M R} .
$$

While there is only a single mass excitation for the branched polymer model and it from this point of view seems rather trivial, the $2 \mathrm{~d}$ gravity model seems to contain an infinite tower of equidistant mass excitations. Since we get the susceptibility $\chi\left(\mu_{r}\right)$ by integrating $G_{M}(R)$ with respect to $R$ we get the following formal expression $\bigoplus$ for $\chi\left(\mu_{r}\right)$

$$
Z^{\prime \prime}\left(\mu_{r}\right)=\chi\left(\mu_{r}\right)=\frac{1}{2} M^{2} \sum_{n=1}^{\infty} n \quad\left(=-\frac{1}{6} \mu_{r}^{1 / 2}\right),
$$

where the last equality sign uses $\sum_{n=1}^{\infty} n=-1 / 12$. It agrees of course with the universal part of (60) but is interesting since integration (ignoring non-universal parts of $\left.Z\left(\mu_{r}\right)\right)$ leads to the following representation of $Z\left(\mu_{r}\right)$ as a sum over "mass excitations":

$$
Z\left(\mu_{r}\right)=\frac{4}{3} \mu_{r}^{9 / 4} \sum_{n=1}^{\infty} n M
$$

\section{4 discussion}

We have shown that the 2-point function $G_{\mu}(r)$ is a natural variable which allows us to extract scaling relations in a simple way. We tested the procedure in $2 \mathrm{~d}$ quantum gravity which can be solved analytically and found $\nu=1 / 4$ and $\eta=4$. From the scaling relations (13) and (14) we conclude that $\gamma=-1 / 2$ (which is of course well known) and $d_{H}=4$. At first sight it might be surprising that the Hausdorff dimension is 4 , which implies that the geodesic distances scale like $r \sqrt{a}$ rather than like $a r$, where $a$ is the lattice spacing in the triangulations. However, such non-trivial scaling is unavoidable if $d_{H} \neq 2$ and here it has the following simple interpretation: A boundary of $l$ links will have the discrete length $l$ in lattice units, but if we view the boundary from the interior of the surface its true linear extension $r$ will only be $\sqrt{l}$ since the boundary can be viewed as a random walk from the interior. If we insist on a continuum limit where we have surfaces with macroscopic boundaries of length $L=a l$ and "physical" area $A=N a^{2}$, a being the length unit of the links, such that $L^{2} \sim A$, we are led to

$$
A \sim L^{2} \sim a^{2} l^{2} \sim a^{2} r^{4} \sim R^{4} .
$$

\footnotetext{
${ }^{5}$ The divergence of the sum comes from the short distance behavior of $G_{M}(R)$. In the discretized version of the theory this singular behavior is modified for $R \sim 1 / \sqrt{a}$ and a "physical cut off" would be present such that

$$
\chi\left(\mu_{r}\right)=\frac{1}{2} M^{2} \sum_{n} n \mathrm{e}^{-n M \sqrt{a}}=\frac{1}{2 a}-\frac{1}{6} \sqrt{\mu_{r}}
$$
}

in agreement with 60 ). 
The above calculation is independent of the fact that we used triangulations as the underlying discretization. Any resonable distribution of polygons will give the same results. Once the distribution of polygons is fixed there is a unique critical point $\mu_{c}$ of the chemical potential for polygons. The discretized equation will still be given by (37), only will $f(x)$ be a higher order (even infinite order) polynomium times a square root cut. Close to the critical point it will still maintain the structure (40) as follows from the general analysis of matrix models [17 and the results will be unchanged for $\Delta \mu \rightarrow 0$. In particular this shows that we would have obtained the same results if we used the shortest link length as geodesic distance, rather than the shortest link length on the dual lattice, since we could instead perform the summation over $\phi^{3}$ graphs. These would be in one-to-one correspondence with the triangulations and our definition of geodesic distance on this class of graphs would correspond to the link-length definition on the triangulations.

We find formula (64) quite interesting. It should be possible to understand the mass excitations $n M$ in terms of Liouville theory.

It is not clear that the program will work well in the case of the so-called multicritical matrix models. In these models the different polygons used in the discretization are not assigned a positive weight in the summation and the basic inequalities like (6) are not valid. In the Liouville formulation the multicritical models correspond the non-unitary conformal field theories coupled to $2 \mathrm{~d}$ quantum gravity and we will face the problem of correlation functions growing with distance. This problem is currently under investigation. In the case of unitary models coupled to gravity we expect our philosophy to apply. These models usually have a representation at the discretized level as short range interacting spin models which at specific temperatures become critical. For these models there is a chance that estimates like (6) might be valid. The standard example is the Ising model on dynamical triangulations. We hope to be able to solve this model by the technique outlined above.

Finally it would be very interesting to generalize the above calculations to higher dimensional simplicial quantum gravity. Work in this direction is in progress [18.

Acknowledgment It is a pleasure to thank Jerzy Jurkiewicz for many interesting discussions.

\section{References}

[1] F. David, Mod.Phys.Lett. A3 (1988) 1651; J. Distler and H. Kawai, Nucl.Phys. B321 (1989) 509.

[2] F. David, Nucl.Phys. B257 (1985) 45; Nucl.Phys. B257 (1985) 543.

[3] J. Ambjørn, B. Durhuus and J. Fröhlich, Nucl.Phys. B257 (1985) 433; B270 (1986) 457; B275 (1986) 161-184.

[4] J. Ambjørn, B. Durhuus J. Fröhlich and P. Orland, B270 (1986) 457; B275 (1986) 161. 
[5] V.A. Kazakov, I. Kostov and A.A. Migdal, Phys.Lett. B157 (1985) 295; Nucl.Phys. B275 (1986) 641.

[6] N. Kawamoto, Y. Saeki, and Y. Watabiki, in preparation; see also N. Kawamoto, INS-Rep.972; Y. Watabiki, Prog.Theor.Phys.Suppl. No.114 (1993) 1.

[7] H. Kawai, N. Kawamoto, T. Mogami and Y. Watabiki, Phys.Lett.B306 (1993) 19.

[8] Y. Watabiki, Construction of Non-critical String Field Theory by Transfer Matrix Formalism in Dynamical Triangulation, INS-Rep.1017, to be published in Nucl. Phys. B.

[9] B. Durhuus, J. Fröhlich and T. Jonsson, Nucl.Phys. B240 (1984) 453; B257 (1985) 779 .

[10] J. Ambjørn, B. Durhuus and T. Jonsson, Mod.Phys.Lett. A6 (1991) 1133.

[11] J. Ambjørn, B. Durhuus and T. Jonsson, Phys.Lett. B244 (1990) 403.

[12] J. Ambjørn and J. Jurkiewicz, Phys.Lett B278 (1992) 42.

[13] M. Agishtein amd A.A. Migdal, Mod.Phys.Lett A7 (1992) 1039.

[14] F. David, Mod.Phys.Lett. A5 (1990) 1019.

[15] J. Ambjørn and Yu.M. Makeenko, Mod.Phys.Lett. A5 (1990) 1753.

[16] S.S. Gubser and I.R. Klebanov, Nucl.Phys. B416 (1994) 827.

[17] J. Ambjørn, L. Chekhov, C.F Kristjansen and Yu. Makeenko, Nucl.Phys. B404 (1993) 127.

[18] J. Ambjørn and J. Jurkiewicz, Scaling in four-dimensional simplicial quantum gravity, to appear. 